\title{
Composition and structure of the shallow subsurface of Ceres revealed by crater morphology
}

\author{
Michael T. Bland ${ }^{1 \star}$, Carol A. Raymond ${ }^{2}$, Paul M. Schenk ${ }^{3}$, Roger R. Fu ${ }^{4}$, Thomas Kneissl ${ }^{5}$, \\ Jan Hendrik Pasckert ${ }^{6}$, Harry Hiesinger ${ }^{6}$, Frank Preusker ${ }^{7}$, Ryan S. Park ${ }^{2}$, Simone Marchi ${ }^{8}$, \\ Scott D. King ${ }^{9}$, Julie C. Castillo-Rogez ${ }^{2}$ and Christopher T. Russell ${ }^{10}$
}

Before NASA's Dawn mission, the dwarf planet Ceres was widely believed to contain a substantial ice-rich layer below its rocky surface. The existence of such a layer has significant implications for Ceres's formation, evolution, and astrobiological potential. Ceres is warmer than icy worlds in the outer Solar System and, if its shallow subsurface is ice-rich, large impact craters are expected to be erased by viscous flow on short geologic timescales. Here we use digital terrain models derived from Dawn Framing Camera images to show that most of Ceres's largest craters are several kilometres deep, and are therefore inconsistent with the existence of an ice-rich subsurface. We further show from numerical simulations that the absence of viscous relaxation over billion-year timescales implies a subsurface viscosity that is at least one thousand times greater than that of pure water ice. We conclude that Ceres's shallow subsurface is no more than $30 \%$ to $40 \%$ ice by volume, with a mixture of rock, salts and/or clathrates accounting for the other $60 \%$ to $70 \%$. However, several anomalously shallow craters are consistent with limited viscous relaxation and may indicate spatial variations in subsurface ice content.

A lthough Ceres' surface is silicate-rich ${ }^{1-3}$, its low density $\left(2,162 \mathrm{~kg} \mathrm{~m}^{-3}\right.$ (ref. 4) from NASA's Dawn mission $\left.{ }^{5}\right)$ combined with measurements of its shape (that is, the difference between its equatorial and polar radius, $a-c)^{6}$ previously suggested that the dwarf planet is differentiated with a rocky core overlain by a relatively pure-ice layer $50-100-\mathrm{km} \mathrm{thick}^{7}$. In this model the rocky surface is probably a lag deposit representing the top of a thin $(\sim 1-10 \mathrm{~km})$, mixed ice-rock layer that avoided complete melting during differentiation ${ }^{8}$, although such a layer may be unstable to disruption by impacts and Rayleigh-Taylor overturn $^{7,9}$. Alternatively, Ceres' interior has been modelled as an ice-free, porous chondritic assemblage ${ }^{10}$, although the thermal stability of such a model is uncertain ${ }^{11}$. Dawn's Visible and Infrared Spectrometer (VIR) has not detected widespread occurrences of water ice on Ceres' surface ${ }^{2}$, where it is not thermodynamically stable ${ }^{12}$. The ice content of the deeper interior remains unknown, and has significant implications for Ceres' formation and subsequent evolution. An ice-rich subsurface would have played a dominant role in shaping Ceres' surface morphology. Ceres is warm when compared to the icy worlds of the outer Solar System (for example, $\sim 50-60 \mathrm{~K}$ warmer than Europa), with diurnally averaged equatorial (latitudes $<30^{\circ}$ ) temperatures of $150-160 \mathrm{~K}$, mid-latitude $\left(\sim 50^{\circ}\right)$ temperatures of $\sim 140 \mathrm{~K}$, and polar $\left(\sim 70^{\circ}\right)$ temperatures of $\sim 120 \mathrm{~K}$ or less ${ }^{12}$. At such warm surface temperatures, ice is not strong enough to retain large-scale topography, and impact craters are expected to undergo viscous relaxation on short timescales ${ }^{13}$. The arrival of the Dawn mission ${ }^{5}$ at Ceres has shown the surface to generally be heavily cratered, including numerous craters with diameter $D \sim 100 \mathrm{~km}$ (ref. 14; Supplementary Fig. 1). The mere existence of these craters, many of which are at low latitudes (that is, warm surface temperatures), belies predictions of a topographically 'smooth', ice-rich Ceres ${ }^{13}$. Here we compare the depths of these largest craters with numerical simulations of viscous relaxation to constrain the physical composition of Ceres' outermost layer.

\section{Measurement of crater depths on Ceres}

We measured the apparent depths $\left(d_{\mathrm{a}}\right)$ of Ceres' largest, clearly identifiable craters (see ref. 15) using a digital terrain model (DTM) derived from Dawn Framing Camera (FC) images with a resolution of $140 \mathrm{~m} \mathrm{pixel}^{-1}$ (from Dawn's High Altitude Mapping Orbit) using stereo-photogrammetry (SPG) methods ${ }^{16}$. The DTM has a mean vertical error of $12 \mathrm{~m}$ and a horizontal resolution similar to the image resolution $(140 \mathrm{~m})$. Because viscous relaxation is wavelength dependent, large craters provide the most stringent constraints on the viscosity required to maintain crater topography (in an infinite half space, large craters relax fastest). We therefore focus on craters with diameters between $75 \mathrm{~km}$ and $280 \mathrm{~km}$, and for wellpreserved craters we consider our inventory complete to diameters above $80 \mathrm{~km}$ ( $n=25$ craters, Supplementary Fig. 1). Apparent depth (that is, depth relative to the surrounding terrain) rather than the rim depth $\left(d_{\mathrm{r}}\right)$ is used because the surrounding terrain

\footnotetext{
${ }^{1}$ US Geological Survey, Astrogeology Science Center, Flagstaff, Arizona 86001, USA. ${ }^{2}$ Jet Propulsion Laboratory, California Institute of Technology, Pasadena, California 91109, USA. ${ }^{3}$ Lunar and Planetary Institute, Houston, Texas 77058, USA. ${ }^{4}$ Columbia University, New York, New York 10027, USA.

${ }^{5}$ Institute of Geological Sciences, Freie Universität Berlin, Berlin 12249, Germany. ${ }^{6}$ Institut für Planetologie, Westfälische Wilhelms-Universität, Münster 48149, Germany. ${ }^{7}$ German Aerospace Center (DLR), Berlin 12489, Germany. ${ }^{8}$ Southwest Research Institute, Boulder, Colorado 80302, USA.

${ }^{9}$ Virginia Institute of Technology, Blacksburg, Virginia 24061, USA. ${ }^{10}$ Department of Earth and Space Sciences, University of California, Los Angeles, California 90095, USA. *e-mail: mbland@usgs.gov
} 


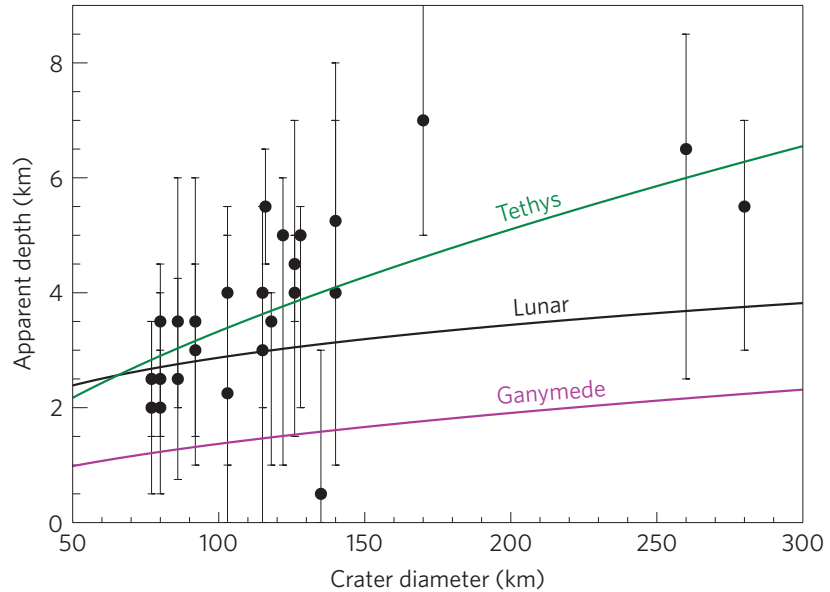

Figure 1 | The apparent depths of Ceres' largest craters. Black dots indicate the characteristic depth of each crater. Vertical bars indicate the azimuthal variability of the depth due to variations in the elevation of the surrounding terrain (see Methods). Curves for fresh craters on Tethys (green) ${ }^{17}$, Ganymede (purple) ${ }^{17}$ and the Moon (black) ${ }^{18}$ are shown for comparison. The curves have been adjusted to account for crater rims.

provides the zero-potential surface to which the crater will relax (for example, a fully relaxed crater can have $d_{\mathrm{a}}=0$, but $d_{\mathrm{r}} \neq 0$ ). Crater depths were measured by comparing the elevation of the floor to a characteristic elevation for the surrounding terrain (see Methods and Supplementary Information).

Our measured crater depths are shown in Fig. 1. Ceres' topography is highly irregular over short spatial scales. The elevation of terrain surrounding each crater can therefore differ substantially in one azimuthal direction compared to another (see Supplementary Methods). This variability is encapsulated in the vertical bars associated with each point in Fig. 1. These bars should not be construed as formal or informal error bars. Rather each point represents the characteristic depth of each crater, and the bars represent the azimuthal variability in the depth measurement (see Methods).

All but one of Ceres' large craters have $d_{\mathrm{a}} \geq 2 \mathrm{~km}$, and seven (Kerwan, Yalode, Urvara, Vinotonus, Zadeni, Chaminuka and Ezinu) have $d_{\mathrm{a}} \geq 5 \mathrm{~km}$. These seven craters range in diameter from $116 \mathrm{~km}$ to $280 \mathrm{~km}$. Only one crater has a characteristic apparent depth of less than $2 \mathrm{~km}$ : Coniraya $\left(40^{\circ} \mathrm{N}, D=135 \mathrm{~km}, d_{\mathrm{a}}=500 \mathrm{~m}\right.$ Supplementary Fig. 7). This crater is discussed in detail below. Many of Ceres' deep craters appear fairly young (for example, Supplementary Figs 3-5, 9 and 11); however, the crater Vinotonus $(D=140 \mathrm{~km})$ is superposed by numerous interior craters, including a 46-km-diameter crater that has destroyed a portion of its southern rim. The high density of superposed craters on Vinotonus indicates that crater topography can be maintained over long timescales. Crater depths are generally consistent with the depth of craters on Saturn's ice-rich satellite Tethys ${ }^{17}$, which has a similar surface gravity to Ceres but whose surface temperature is $\sim 86 \mathrm{~K}$. Craters on Ceres are generally deeper than those on Ganymede and Earth's Moon ${ }^{18,19}$.

\section{The composition of the shallow subsurface}

The depths of Ceres' largest craters are not consistent with the existence of an ice-dominated subsurface composition. Figure 2 shows the measured depths of Ceres' craters compared to finite element simulations of viscous relaxation of crater topography in a pure-ice layer as a function of latitude (surface temperature), crater formation time, and ice grain size. The simulations follow the methodology of ref. 13 (see Methods), assume a 100-km-diameter crater that is initially $4.5-\mathrm{km}$ deep (based on the depths of similar sized fresh craters like Ezinu and Dantu), formation times of $1 \mathrm{Gyr}$

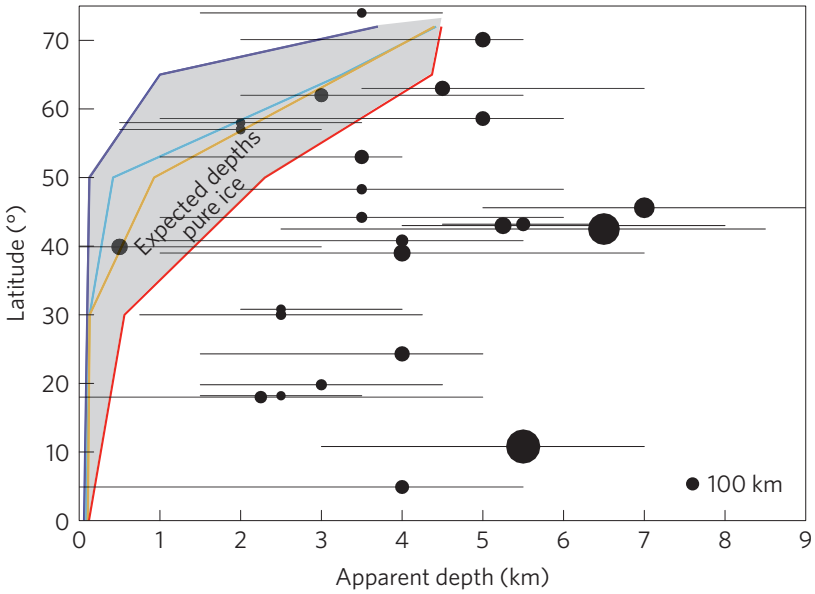

Figure 2 | Comparison of the observed depths of Ceres' largest craters with the depths expected for an ice-rich subsurface composition as a function of latitude. Simulations assume a $100-\mathrm{km}$-diameter crater. Line colour corresponds to different combinations of ice grain size and crater age: $1 \mathrm{~mm}, 1 \mathrm{Gyr}$ (purple); $1 \mathrm{~mm}, 100 \mathrm{Myr}$ (blue); $10 \mathrm{~mm}, 1 \mathrm{Gyr}$ (orange); and $10 \mathrm{~mm}, 100 \mathrm{Myr}$ (red). Symbol size shows crater diameter relative to $100 \mathrm{~km}$, and the horizontal black lines associated with each circle indicate azimuthal variations in crater depth.

ago and $100 \mathrm{Myr}$ ago, and grain sizes of $1 \mathrm{~mm}$ and $10 \mathrm{~mm}$ (ref. 20). Except for Kerwan, Yalode and Urvara $(D=280,260$ and $170 \mathrm{~km})$, the craters included in this investigation are within $40 \%$ of the diameter of the simulated crater. The uncertainty introduced by the use of a generic crater diameter in our simulations is not significant compared to the poorly constrained grain size and crater formation time.

Because of the strong temperature dependence of ice rheology (for example, ref. 21), viscous relaxation is expected to produce distinct latitudinal variations ${ }^{13}$. The measured crater depths shown in Fig. 2 demonstrate that this is not the case. Our pure-ice simulations indicate that at latitudes $\leq 30^{\circ}\left(T_{\mathrm{s}} \sim 150-160 \mathrm{~K}\right)$, viscous relaxation in a pure-ice layer would result in presentday apparent crater depths of less than $500 \mathrm{~m}$, even if ice grains are large (that is, maximizing the ice viscosity) and the craters formed recently (100 Myr ago). At these surface temperatures, a $100-\mathrm{km}$-diameter crater can fully relax $\left(d_{\mathrm{a}} \sim 0\right)$ on timescales of 1-10 Myr. There are six craters with $D>75 \mathrm{~km}$ in this latitude band on Ceres (Fig. 2). All have a characteristic $d_{\mathrm{a}} \geq 2 \mathrm{~km}$, which is much deeper than expected for a pure-ice layer. The crater Dantu ( $D=126 \mathrm{~km}$, Supplementary Fig. 9) at $24^{\circ} \mathrm{N}$, for example, has $d_{\mathrm{a}}=4 \mathrm{~km}$. At higher latitudes $\left(30^{\circ}-50^{\circ}\right)$ our simulations for a pureice layer indicate that crater topography will still viscously relax on timescales of 10-100 Myr, resulting in crater depths of less than $500 \mathrm{~m}$ for a nominal grain size of $1 \mathrm{~mm}$. Craters can retain their topography at these latitudes only if the ice grains are large $(10 \mathrm{~mm}$, increasing the ice viscosity by a factor of $\sim 25$ ) and the craters formed recently. Even then, present-day crater depths of just 1-2 km are expected (red curve in Fig. 2). In contrast, numerous craters with $d_{\mathrm{a}}$ exceeding $2 \mathrm{~km}$ are observed in this latitude range (Fig. 2). These craters include four of the five deepest on the dwarf planet: Urvara, Yalode, Vinotonus and Ezinu.

Ceres' polar regions are cold enough to retain crater topography over long timescales even if the subsurface is pure ice. Notably, the presumably well-preserved polar craters, such as the $128-\mathrm{km}$-diameter Zadeni $\left(70^{\circ} \mathrm{S}\right)$, have nearly equivalent depths $(5 \mathrm{~km})$ to similarly sized craters at lower latitudes, such as the $140-\mathrm{km}$-diameter Vinotonus $\left(43^{\circ} \mathrm{N}, 5-\mathrm{km}\right.$ depth) and the 126-km-diameter Dantu ( $24^{\circ} \mathrm{N}, 4-\mathrm{km}$ depth). Systematic latitudinal variations in crater depths are not observed on Ceres; however, 


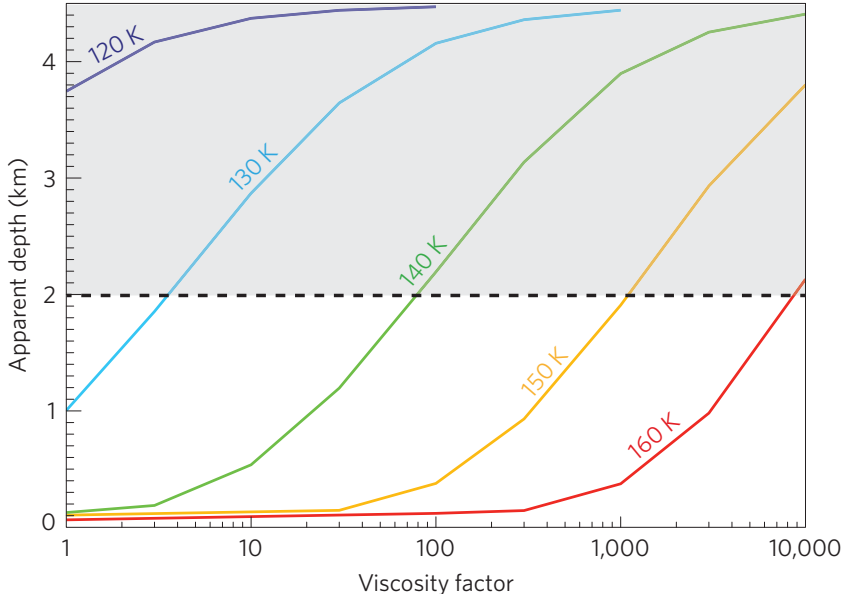

Figure 3 | The subsurface viscosity required to preserve crater topography on Ceres. Coloured curves show the simulated present-day apparent depth of a $100-\mathrm{km}$-diameter crater formed $1 \mathrm{Gyr}$ ago (initially $4.5-\mathrm{km}$ deep) as a function of viscosity increase relative to water ice and surface temperature. Surface temperatures $\left(T_{\mathrm{s}}\right)$ of $150-160 \mathrm{~K}$ (red, orange) correspond to latitudes $<30^{\circ}$, whereas $T_{\mathrm{s}}=140 \mathrm{~K}, 130 \mathrm{~K}$ and $120 \mathrm{~K}$ (green, blue and violet) correspond to latitudes of $50^{\circ}, 66^{\circ}$ and $72^{\circ}$, respectively ${ }^{12}$. The grey box indicates the region of typical crater depths on Ceres.

variations in topographic relaxation were identified on Ceres in ref. 22 at wavelengths generally longer than (and so more susceptible to relaxation) those investigated here (see discussion below).

To constrain the viscosity required to preserve impact crater topography, we performed numerical simulations of crater relaxation in which we systematically increase the viscosity (relative to water ice) of Ceres' subsurface. The simulated present-day depth of a $100-\mathrm{km}$ diameter, $4.5-\mathrm{km}$-deep crater is shown in Fig. 3 as a function of surface temperature and viscosity factor (that is, the increase relative to pure water ice). At equatorial and mid-latitude temperatures of $150-160 \mathrm{~K}$, increasing the viscosity by a factor of 100 is insufficient to retain crater topography. For these surface temperatures, only a viscosity increase of $\geq 10^{3} \times$ is sufficient to retain crater depths of greater than $2 \mathrm{~km}$. At higher latitudes, where surface temperatures are lower $(\sim 130 \mathrm{~K})$, viscosity increases on the order of a factor of ten are sufficient to retain topography.

An increase in the viscosity of Ceres' outer shell sufficient to maintain impact crater topography for $1 \mathrm{Gyr}$ or longer requires the presence of a volumetrically substantial non-ice component in the subsurface. Theoretical modelling ${ }^{23,24}$ and laboratory experiments $^{25,26}$ indicate that particulate volume fractions $\left(\varphi_{\mathrm{p}}\right)$ of $\geq 60 \%$ are required to increase the viscosity of an ice mixture by a factor of 100 or more. Experiments in sand-ice mixtures have shown that the transition from ductile (if retarded) behaviour to essentially complete immobility occurs over a very narrow range of $\varphi_{\mathrm{p}}(\sim 5 \%)^{26}$. The required $\varphi_{\mathrm{p}}$ for this 'jammed' behaviour depends on the distribution of grain sizes, but appears to be higher ( $\sim 70 \%$ ) for non-uniform grain sizes, which are likely to be more representative of Ceres' outer layer ${ }^{26,27}$. Ceres' outer layer is therefore probably relatively ice poor, with non-ice material constituting $60-70 \%$ of the volume. This result is consistent with independent inferences of the viscosity structure of Ceres from its global shape $e^{22}$.

A range of plausible compositions for Ceres' outer layer are permitted by its weakly constrained internal structure ${ }^{4}$ and surface composition $^{2,3}$. That Ceres' interior is centrally condensed ${ }^{4}$ suggests that the outer layer must have a density lower than Ceres' average density of $2,162 \mathrm{~kg} \mathrm{~m}^{-3}$. If Ceres' outer layer is simply a combination of silicate material $\left(\rho_{\mathrm{s}}=2,500 \mathrm{~kg} \mathrm{~m}^{-3}\right.$, probably a minimum silicate density) and ice, the $60 \%$ minimum $\varphi_{\mathrm{p}}$ required to maintain crater topography dictates a subsurface density in excess of
$1,800 \mathrm{~kg} \mathrm{~m}^{-3}$ (resulting in a relatively thick outer layer). Particulate fractions of $\sim 70 \%$ are excluded by the density constraints if a two-component ice-silicate mixture is assumed (that is, the resulting density is greater than Ceres' mean density). However, the addition of strong, but low-density material such as salts (hydrated or anhydrous) would have a similar effect to that of rock on ice rheology (ref. 28 demonstrated this in the dislocation creep regime, and it is probably true for other flow mechanisms, although laboratory data are sparse). Low-density clathrates are also $\sim 100-1,000$ times stronger than water ice at Ceres' temperatures (depending on composition and extrapolations from laboratory data taken at warmer temperatures $)^{29}$. Carbonates ${ }^{30}$ and salts ${ }^{31}$ of internal origin have been detected at Occator ${ }^{30}$ and the occurrence of methane clathrates is predicted from geochemical models (J. C. Castillo-Rogez, personal communication). The inclusion of these (or similar) low-density materials in Ceres' subsurface permits a broader range of outer layer densities (and hence, thicknesses). Ceres' subsurface is therefore more likely to be composed of a combination of ice, rock, and a strong, low-density material, with hydrated salts and/or clathrates as possible candidates.

\section{Evidence for limited viscous relaxation}

Despite the predominance of deep craters on Ceres, a few large craters are anomalously shallow and may provide evidence for lateral variations in the ice content of Ceres' outer layer (Fig. 4). The neighbouring and similarly sized craters Coniraya and Vinotonus (both $D \approx 135-140 \mathrm{~km}$ ) have vastly different apparent depths $\left(d_{\mathrm{a}}\right.$ of $500 \mathrm{~m}$ and $5.25 \mathrm{~km}$, respectively). Both craters appear to be of similar age, based on superposed crater densities (Supplementary Fig. 2), and both have probably retained their respective morphologies for a long time. Coniraya does not appear to be obviously filled by crater ejecta from either Vinotonus or the nearby crater Gaue: ejecta from Vinotonus at the centre of Coniraya would have a depth of just $\sim 250 \mathrm{~m}$, assuming ejecta thickness decreases as $R^{-3}$ (ref. 32). Coniraya is therefore one of the best candidates for a crater on Ceres that has undergone viscous relaxation. Relaxation of a Vinotonus-like initial crater would result in Coniraya-like topography in $\sim 1 \mathrm{Gyr}$ if $\varphi_{\mathrm{p}}$ is generally near the 'jammed' transition but is $10-20 \%$ lower beneath Coniraya (for example, $\varphi_{\mathrm{p}} \sim 70 \%$ beneath Vinotonus and $50-60 \%$ beneath Coniraya). This scenario is non-unique as there is a tradeoff between shorter relaxation times and lower values of $\varphi_{\mathrm{p}}$. Alternatively, we cannot rule out the possibility that Coniraya formed from a large, very-low-velocity impact that produced a similar diameter but much shallower crater than Vinotonus, but we do not favour this explanation. To first order, $d / D$ is independent of impact velocity: the transient crater is generally parabolic, so depth and diameter scale together ${ }^{32}$.

The large, equatorial crater Kerwan is also anomalous. The crater is fairly deep $(5.5 \mathrm{~km})$, but rather than having steep rims and a flat floor, as is typical for craters of its size, its rim is a subtle topographic step, and the crater floor gradually deepens from crater rim to centre (Fig. 4 and Supplementary Fig. 3). Its topography differs substantially from that of Yalode and Urvara, Ceres' next largest craters, although, unlike Vinotonus and Coniraya, the age and surface temperatures of these three craters differ substantially. The morphology is consistent with viscous relaxation in a thin, low-viscosity layer underlain by an immobile layer at $\sim 30-\mathrm{km}$ $\operatorname{depth}^{33}$. Kerwan might then provide evidence for a locally thin outer layer, an inference which may be evaluated by future Dawn gravity measurements. Notably, Kerwan is the largest crater on Ceres, and given its location (at the warm equator) and its size, it is the crater most expected to have undergone relaxation on Ceres. A viscously relaxed Kerwan is also consistent with observations of moderate relaxation in Ceres' equatorial region observed at long wavelengths ${ }^{22}$. 

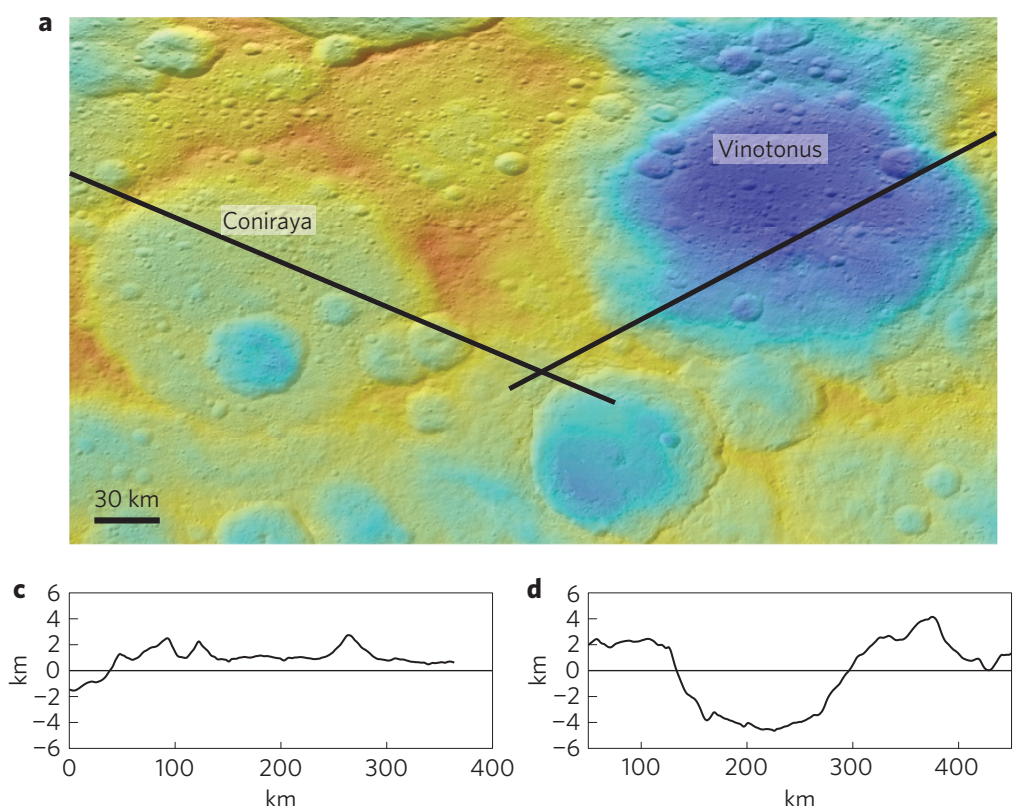
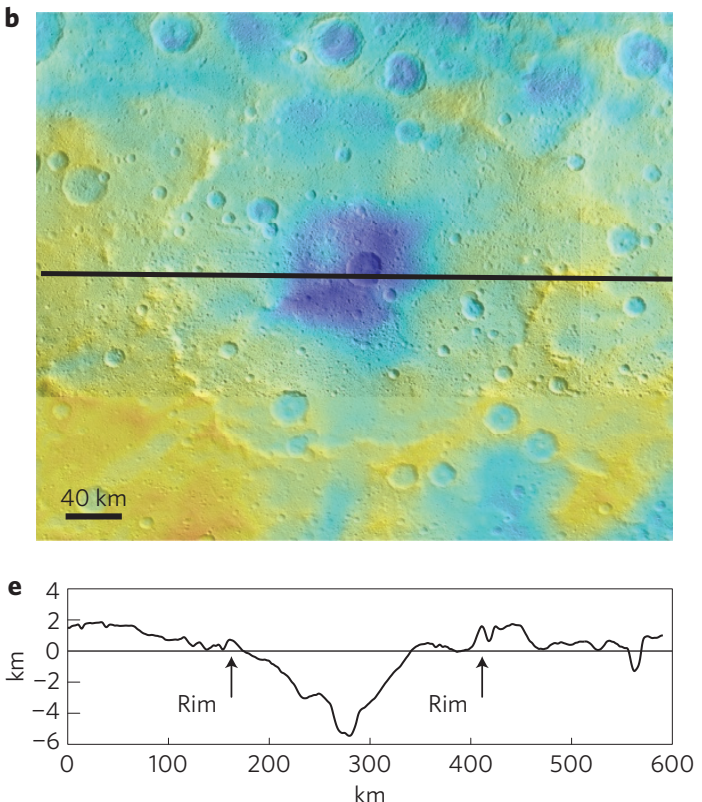

Figure 4 | The topography of craters on Ceres that exhibit possible evidence for viscous relaxation. a,c,d, A comparison of craters Coniraya and Vinotonus (a), with topographic profiles along the black lines for Coniraya and Vinotonus shown in $\mathbf{c}$ and $\mathbf{d}$, respectively. b,e, The crater Kerwan (b) with an east to west profile along the black line shown in e. Note that the profile passes through the crater Insitor near the centre of Kerwan. Colours indicate elevations ranging from $-5,000 \mathrm{~m}$ (blue) to $+5,000 \mathrm{~m}$ (red).

Viscous relaxation of Coniraya (and plausibly Kerwan) can probably only be achieved if the ice content $\left(\varphi_{\mathrm{i}}\right)$ in the subsurface near these craters is enhanced relative to the rest of Ceres (that is, $\left.\varphi_{\mathrm{i}}>40 \%\right)$. However, because the transition from immobile to mobile ice-rock mixtures occurs abruptly as $\varphi_{\mathrm{i}}$ decreases $^{26}$, the corresponding variation in volume fraction may be small. The observation that most of Ceres' craters have retained their topography over long timescales, whereas a few craters have undergone relaxation, indicates that Ceres' outer layer is geologically complex. Both the one-dimensional endmember models of refs 7,10 must therefore be abandoned in favour of interior and evolution models that predict more compositionally heterogeneous and spatially varying interior structures.

\section{Methods}

Methods, including statements of data availability and any associated accession codes and references, are available in the online version of this paper.

\section{Received 9 March 2016; accepted 18 May 2016;} published online 29 June 2016

\section{References}

1. Rivkin, A. S. et al. The surface composition of Ceres. Space Sci. Rev. 163, 95-116 (2011).

2. De Sanctis, M. C. et al. Ammoniated phyllosilicates with a likely outer Solar System origin on (1) Ceres. Nature 528, 241-244 (2015).

3. Ammannito, E. et al. The distribution of phyllosilicates on Ceres. Lunar Planet. Sci. Conf. 47, 3020 (2016).

4. Park, R. S. et al. Gravity science investigation of Ceres from Dawn. Lunar Planet. Sci. Conf. 47, 1781 (2016).

5. Russell, C. T. \& Raymond, C. A. The dawn mission to Vesta and Ceres. Space Sci. Rev. 163, 3-23 (2011).

6. Thomas, P. C. et al. Differentiation of the asteroid Ceres as revealed by its shape. Nature 437, 224-226 (2005).

7. Castillo-Rogez, J. C. \& McCord, T. B. Ceres evolution and present state constrained by shaped data. Icarus 205, 443-459 (2010).

8. McCord, T. B. \& Sotin, C. Ceres: evolution and current state. J. Geophys. Res. 110, E05009 (2005)

9. Roberts, J. H. \& Rivkin, A. S. The fate of Ceres' original crust. AAS Div. Planet. Sci. Conf. 47, 212.07 (2015).
10. Zolotov, M. Y. On the composition and differentiation of Ceres. Icarus 204, 183-193 (2009).

11. Castillo-Rogez, J. C. Ceres-Neither a porous nor salty ball. Icarus 215, 599-602 (2011).

12. Hayne, P. O. \& Aharonson, O. Thermal stability of ice on Ceres with rough topography. J. Geophys. Res. 120, 1567-1584 (2015).

13. Bland, M. T. Predicted crater morphologies on Ceres: probing internal structure and evolution. Icarus 226, 510-521 (2013).

14. Schenk, P. M. et al. Impact cratering on the small planets Ceres and Vesta: S-C transitions, central pits and the origin of the bright spots. Lunar Planet. Sci. Conf. 47, 2697 (2016).

15. Marchi, S. et al. Cratering on Ceres: the puzzle of the missing large craters. Lunar Planet Sci. Conf. 47, 1281 (2016).

16. Raymond, C. A. et al. The Dawn topography investigation. Space Sci. Rev. 163, 487-510 (2011).

17. White, O. L. \& Schenk, P. M. Crater shapes on the saturnian satellites: new measurements using Cassini stereo images. Lunar Planet Sci. Conf. 42, 2283 (2011).

18. Bray, V. J., Collins, G. S., Morgan, J. V. \& Schenk, P. M. The effects of target properties on crater morphology: comparison of central peak craters on the Moon and Ganymede. Meteorit. Planet. Sci. 43, 1979-1992 (2008).

19. Pike, R. J. in Impact and Explosion Cratering (eds Roddy, D. J., Pepin, R. O. \& Merill, R. B.) 489-509 (Pergamon, 1977).

20. Barr, A. C. \& McKinnon, W. B. Convection in ice I shells and mantles with self-consistent grain size. J. Geophys. Res. 112, E02012 (2007).

21. Durham, W. B. \& Stern, L. A. Rheological properties of water ice-Applications to satellites of the outer planets. Annu. Rev. Earth Planet. Sci. 29, 295-330 (2001).

22. Ermakov, A. I. et al. Evaluation of Ceres' compensation state. Lunar Planet. Sci. Conf. 47, 1708 (2016).

23. Roscoe, R. The viscosity of suspensions of rigid spheres. Br. J. Appl. Phys. 3, 267-269 (1952).

24. Friedson, A. J. \& Stevenson, D. J. Viscosity of rock-ice mixtures and applications to the evolution of the icy satellites. Icarus 56, 1-14 (1983).

25. Durham, W. B., Kirby, S. H. \& Stern, L. A. Effects of dispersed particulated on the rheology of water ice at planetary conditions. J. Geophys. Res. 97, 20883-20897 (1992).

26. Durham, W. B., Pathare, A. V., Stern, L. A. \& Lenferink, H. J. Mobility of icy sand packs, with application to Martian permafrost. Geophys. Res. Lett. 36, L23203 (2009).

27. Mangold, N., Allemand, P., Duval, P., Geraud, Y. \& Thomas, P. Experimental and theoretical deformation of ice-rock mixtures: implications on rheology and ice content of Martian permafrost. Planet. Space. Sci. 50, 385-401 (2002). 
28. McCarthy, C., Cooper, R. F., Goldsby, D. L., Durham, W. B. \& Kirby, S. H. Transient and steady state creep response of ice I and magnesium sulfate hydrate eutectic aggregates. J. Geophys. Res. 116, E04007 (2011).

29. Durham, W. B., Prieto-Ballesteros, O., Goldsby, D. L. \& Kargel, J. S. Rheology and thermal properties of icy material. Space Sci. Rev. 153, 273-298 (2010).

30. De Sanctis, M. C. et al. Bright carbonate deposits as evidence of aqueous alteration on (1) Ceres. Nature http://dx.doi.org/10.1038/nature18290 (2016)

31. Nathues, A. et al. Sublimation in bright spots on (1) Ceres. Nature 528, 237-240 (2015).

32. Melosh, H. J. Impact Cratering: A Geologic Process (Oxford Univ. Press, 1989).

33. Parmentier, E. M. \& Head, J. W. Viscous relaxation of impact craters on icy planetary surfaces: determination of viscosity variation with depth. Icarus 47 , 100-111 (1981).

\section{Acknowledgements}

M.T.B. thanks Trent Hare for ArcGIS support. This work was supported by NASA's Dawn Guest Investigator Program (NNH15AZ85I).

\section{Author contributions}

M.T.B. measured all craters, designed, performed and analysed all simulations, and wrote the manuscript. P.M.S. provided comparison data for crater depths on planetary bodies and discussed the results and implications of the observations and simulations. T.K., J.H.P. and H.H. provided crater counts for Coniraya and Vinotonus and discussed implications of the results. F.P. and R.S.P. created and provided the DTMs used to measure crater depths. C.A.R., R.R.F., J.C.C.-R., S.D.K. and S.M. provided substantive discussion of the observations, simulations and implications for Ceres' near-surface composition, and provided critical comments on the manuscript at all stages of development. C.T.R. is responsible for the Dawn mission and all data acquisition.

\section{Additional information}

Supplementary information is available in the online version of the paper. Reprints and permissions information is available online at www.nature.com/reprints. Correspondence and requests for materials should be addressed to M.T.B.

\section{Competing financial interests}

The authors declare no competing financial interests. 


\section{Methods}

We measured apparent crater depths using 16 elevation profiles for each crater, which were extracted approximately every $22.5^{\circ}$ and used to determine both the elevation of the crater floor and surrounding terrain (Supplementary Figs 3-15). The method is intentionally subjective, allowing for geologic interpretation to inform the inferred elevations, and permits a more direct assessment of the variability in crater depth as a function of azimuth. In general, the elevation of the crater floor can be determined to within a few hundred metres by this method (a few craters have substantial variation across their floors), whereas the elevation of the surrounding terrain often varies by several kilometres. For example, the deepest portions of the floor of the crater Dantu (Supplementary Fig. 9) are consistently at an elevation of $-5 \mathrm{~km}$. The elevation of the surrounding terrain is typically at an elevation of $-1 \mathrm{~km}$, yielding a characteristic depth of $4 \mathrm{~km}$. However, the terrain to the southwest of the crater has an elevation as low as $-3.5 \mathrm{~km}$ (for example, profiles 11-13 in Supplementary Fig. 9), and the terrain to the north and northeast is higher, with an elevation of $+1 \mathrm{~km}$ (for example, profile 1 of Supplementary Fig. 9). The result is a maximum and minimum plausible crater depth of $6 \mathrm{~km}$ and $1.5 \mathrm{~km}$, respectively. Note that portions of profiles that transect adjacent craters were excluded. The same analysis was applied to each crater, and the variations are reflected in the vertical bars accompanying each depth measurement shown in Figs 1 and 2.

Simulations of viscous relaxation follow the methods described in ref. 13. We use the viscoelastic finite element model Tekton2.3 (ref. 34) in an axisymmetric geometry. The bottom boundary is fixed in the vertical and horizontal, and the sides of the domain are free slip in the vertical and fixed in the horizontal. The top of the domain is a free surface. The $100-\mathrm{km}$-diameter crater imposed on the domain was $4.5 \mathrm{~km}$ deep, with a rim height of $1.4 \mathrm{~km}$. The floor of the crater is flat until within $20 \mathrm{~km}$ of the crater rim. For simplicity we do not include a central peak. The crater shape was based on Ceres' well-preserved craters, Ezinu and Dantu. The simulation domain was $200 \mathrm{~km}$ long and $150 \mathrm{~km}$ deep, and had a horizontal resolution of $1 \mathrm{~km}$ and a vertical resolution that varied from $50 \mathrm{~m}$ in the near subsurface to $4 \mathrm{~km}$ at the base of the domain. Stresses were initialized with a combination of lithostatic stress (Ceres' surface gravity is $0.27 \mathrm{~m} \mathrm{~s}^{-2}$ ) and topographic stress following ref. 35 .

Tekton 2.3 does not explicitly include thermodynamics, but the viscosity structure of each simulation is determined by an externally calculated thermal structure, which is allowed to evolve with time. We assume Ceres' heat flux results from time-dependent radiogenic heating, assuming a CI-chondrite complement of radiogenic species ${ }^{13}$. Thus simulations that use a crater formation time of $1 \mathrm{Gyr}$ ago experience both longer durations of heating, and higher maximum heat fluxes compared to simulations that assume a crater formation age of 100 Myr. However, for the timescales considered here, the change in heat flux over the course of a simulation is small. The maximum heat flux in our simulations $1 \mathrm{Gyr}$ ago was $\sim 1.5 \mathrm{~mW} \mathrm{~m}^{-2}$, whereas the present-day heat flux is $\sim 1 \mathrm{~mW} \mathrm{~m}^{-2}$. Note that any process that enriches radiogenic species relative to CI chondrites would lead to more rapid viscous relaxation due to the higher resulting heat fluxes, making an ice-rich composition even less plausible. Because the thermal structure is already nearly isothermal (at present $\mathrm{d} T / \mathrm{d} z \sim 0.2 \mathrm{~K} \mathrm{~km}^{-1}$ ), decreasing the radiogenic component has little effect on our simulations. We examine a range of surface temperatures based on those derived for Ceres in ref. 12.

Our simulations include all relevant flow laws for ice (I), including dislocation creep (three regimes $A, B$ and $C$ ), diffusion (Diff), grain-boundary sliding (GBS), and basal slip $(\mathrm{BS})^{21}$. The flow mechanisms are combined into a composite flow law where the total strain rate $\dot{\varepsilon}_{\text {tot }}$ is given by

$$
\dot{\varepsilon}_{\text {tot }}=\dot{\varepsilon}_{\mathrm{A}}+\dot{\varepsilon}_{\mathrm{B}}+\dot{\varepsilon}_{\mathrm{C}}+\dot{\varepsilon}_{\mathrm{Diff}}+\left[\frac{1}{\dot{\varepsilon}_{\mathrm{GBS}}}+\frac{1}{\dot{\varepsilon}_{\mathrm{BS}}}\right]^{-1}
$$

where each term on the right has the form

$$
\dot{\varepsilon}=A^{*} d_{\mathrm{i}}^{-m} \sigma^{n} \exp \left[\frac{-Q}{R T}\right]
$$

and $A^{*}$ is a rheological constant (here modified from that derived in uniaxial experiments (see ref. 36)), $d_{\mathrm{i}}$ is the ice grain size, $m$ is the grain size exponent, $\sigma$ is the deviatoric stress, $n$ is the power law exponent, $Q$ is the activation energy, $R$ is the gas constant, and $T$ is the temperature. Note that we neglect the pressure dependence, which is typically small. For the low stresses and warm temperatures in our simulations, grain-boundary sliding and diffusion dominate the rheology. Grain-boundary sliding and diffusion are both grain-size-sensitive $(m \neq 0)$. We nominally use a grain size of $1 \mathrm{~mm}$, consistent with models of icy satellite tectonics (see discussion in refs 20,37 ) and terrestrial glaciers ${ }^{38,39}$. We also consider larger grain sizes $(10 \mathrm{~mm})$ to insure that the retention of Ceres' topography cannot be attributed to stiff, large-grained ice, but note that such large grains are inconsistent with the presence of particulate material, which tends to inhibit grain growth ${ }^{20}$.

The inclusion of particulate material with ice (either hard sand $\mathrm{d}^{25-27}$ or salts ${ }^{28}$ ) is known to increase the viscosity of the mixture relative to pure samples. On the basis of laboratory data, the effect of particulates has been parameterized by multiplying the effective viscosity by a factor of $e^{b n \phi_{\mathrm{p}}}$, where $b=2$ is experimentally determined ${ }^{13,21,40,41}$. By this formulation a particulate fraction of $\varphi_{\mathrm{p}}=0.5$ for grain-boundary sliding $(n=1.8)$ results in an approximately factor of six increase in viscosity. However, this relationship breaks down at particulate fractions above $\sim 0.6$ (refs $24-26$ ). For this reason, we simply increase the viscosity by a given factor (for example, by a factor of 100 or 1,000) instead of attempting to model a specific $\varphi_{\mathrm{p}}$.

Code availability. The original Tekton 2.3 source code used to simulate viscous relaxation is no longer publicly available. Contact the corresponding author for additional information.

Data availability. All Dawn FC images used in this study will be made available in NASA's Planetary Data System (PDS) archive (Small Bodies Node:

http://pds-smallbodies.astro.umd.edu) in accordance with Dawn's data release plan.

\section{References}

34. Melosh, H. J. \& Raefsky, A. The dynamical origin of subduction zone topography. Geophys. J. R. Astron. Soc. 60, 333-334 (1980).

35. Dombard, A. J. \& McKinnon, W. B. Elastoviscoplastic relaxation of impact crater topography with application to Ganymede and Callisto. J. Geophys. Res. 111, E01001 (2006).

36. Ranalli, G. The Rheology of the Earth 76-77 (Chapman and Hall, 1995).

37. Bland, M. T. \& McKinnon, W. B. Forming Europa's folds: strain requirements for the production of large-amplitude deformation. Icarus 221, 694-709 (2012).

38. De La Chappelle, S., Castelnau, O., Lipenkov, V. \& Duval, P. Dynamic recrystallization and texture development in ice as revealed by the study of the deep ice cores in Antarctica and Greenland. J. Geophys. Res. 103, 5091-5105 (1998).

39. Souchez, R. A. \& Lorrain, R. D. Ice Composition and Glacier Dynamics (Springer, 1991).

40. Pathare, A. V., Paige, D. \& Turtle, E. Viscous relaxation of craters within the Martian south polar layered deposits. Icarus 174, 396-418 (2005).

41. Sori, M. M., Byrne, S., Hamilton, C. W. \& Landis, M. E. Viscous flow rates of icy topography on the north polar layered deposits of Mars. Geophys. Res. Lett. 43, 3060-3068 (2016). 